\title{
The VISTA Carina Nebula Survey
}

\section{Introduction and source catalog ${ }^{\star}, \star \star$}

\author{
T. Preibisch ${ }^{1}$, P. Zeidler ${ }^{1,2}$, T. Ratzka ${ }^{1,3}$, V. Roccatagliata ${ }^{1}$, and M. G. Petr-Gotzens ${ }^{4}$ \\ 1 Universitäts-Sternwarte München, Ludwig-Maximilians-Universität, Scheinerstr. 1, 81679 München, Germany \\ e-mail: preibisch@usm.uni-muenchen.de \\ 2 Astronomisches Rechen-Institut, Zentrum für Astronomie der Universität Heidelberg, Mönchhofstr. 12-14, 69120 Heidelberg, \\ Germany \\ 3 Institute for Physics/IGAM, NAWI Graz, Karl-Franzens-Universität, Universitätsplatz 5/II, 8010 Graz, Austria \\ ${ }^{4}$ European Southern Observatory, Karl-Schwarzschild-Str. 2, 85748 Garching, Germany
}

Received 22 April 2014 / Accepted 6 October 2014

\section{ABSTRACT}

\begin{abstract}
Context. The Carina Nebula is one of the most massive and active star-forming regions in our Galaxy and has been studied with numerous multiwavelength observations in the past five years. However, most of these studies were restricted to the inner parts ( $\$ 1$ square-degree) of the nebula, and thus covered only a small fraction of the whole cloud complex.

Aims. Our aim was to conduct a near-infrared survey that covers the full spatial extent ( $\sim 5$ square-degrees) of the Carina Nebula complex and is sensitive enough to detect all associated young stars through extinctions of up to $A_{V} \approx 6 \mathrm{mag}$.

Methods. We used the $4 \mathrm{~m}$ Visible and Infrared Survey Telescope for Astronomy (VISTA) of ESO to map an area of 6.7 squaredegrees around the Carina Nebula in the near-infrared $J-, H-, K_{\mathrm{s}}$-bands.

Results. The analysis of our VISTA data revealed 4840807 individual near-infrared sources, 3951580 of which are detected in at least two bands. The faintest $S / N \geq 3$ detections have magnitudes of $J \approx 21.2, H \approx 19.9$, and $K_{\mathrm{s}} \approx 19.3$. For objects at the distance of the Carina Nebula $(2.3 \mathrm{kpc})$, our catalog is estimated to be complete down to stellar masses of $\approx 0.1 M_{\odot}$ for young stars with extinctions of $A_{V} \approx 5 \mathrm{mag}$; for regions in the brightest parts of the central nebula with particularly strong diffuse emission, the completeness limit is at slightly higher stellar masses. We describe the photometric calibration, the characteristics, and the quality of these data. VISTA images of several newly detected or yet rarely studied clusters in the outer parts of the Carina Nebula complex are presented. Finally, a list of stars with high proper motions that were discovered in our analysis is provided in an appendix.

Conclusions. Our catalog represents by far the most comprehensive deep near-infrared catalog of the Carina Nebula complex. It provides a new basis for spatially complete investigations of the young stellar population in this important star-forming complex.
\end{abstract}

Key words. stars: formation - stars: pre-main sequence - open clusters and associations: individual: NGC 3324 -

ISM: individual objects: Gum 31 - ISM: individual objects: NGC 3372

\section{Introduction}

As one of the largest and most prominent H II regions in the sky, the Carina Nebula (NGC 3372) has been the subject of many observational studies since its first scientific description by Sir John F. W. Herschel (Herschel 1847). A recent summary is given in Smith \& Brooks (2008). While the Carina Nebula was famous for a long time for its content of numerous very massive and luminous stars ( $\geq 70$ O-type and Wolf-Rayet (WR) stars; see Smith 2006), including the famous luminous blue variable $\eta \mathrm{Car}$, its nature as one of the most active star-forming regions in our Galaxy was recognized only recently. Until some 15 years ago, the Carina Nebula was generally considered to be an evolved $\mathrm{H}$ II region, lacking any significant amount of ongoing star formation. This impression was partly related to the fact that most observations until that time had concentrated on quite small fields in the central area of the nebula. Smith et al. (2000)

* Based on observations made with ESO Telescopes at the La Silla Paranal Observatory under programme ID 088.C-0117.

$\star \star$ The catalog (full Table 2) is only available at the CDS via anonymous ftp to cdsarc.u-strasbg. fr (130.79.128.5) or via http://cdsarc.u-strasbg.fr/viz-bin/qcat?J/A+A/572/A116 provided an important contribution to the "revolution" in our view of the Carina Nebula; it showed that the central Carina Nebula, which harbors the famous stellar clusters $\operatorname{Tr} 14,15$, and 16 , is surrounded by a large complex of dense clouds that extends over more than $\sim 50 \mathrm{pc}$ and contains numerous embedded infrared sources. This highlighted the importance of spatially complete wide-field surveys to characterize the general properties of H II and star-forming regions.

The high levels of UV radiation in the central parts of the Carina Nebula produce irradiation effects on the surrounding clouds that are on a similar order as seen in extragalactic starburst regions (see Roccatagliata et al. 2013). Furthermore, the combined stellar wind power (see Smith 2006) also profoundly influences the clouds. In the central few pc region of the Carina Nebula, this stellar feedback has already dispersed a large portion of the original dense molecular clouds (see Smith et al. 2010a). At distances of more than a few pc from the massive stars, numerous dense clouds still exist, and the compression of these clouds by the ionization fronts and expanding wind bubbles is currently triggering the formation of new generations of stars in the complex (Smith et al. 2010b; Gaczkowski et al. 2013). Detailed studies of the physical processes in the Carina 
Nebula can thus provide us with important information that is also relevant for the evolution of the (much more distant and thus harder to study) extragalactic starburst regions.

During the past five years, several sensitive surveys of different parts of the Carina Nebula have been performed at wavelengths from the X-ray to the sub-mm regime. One main milestone was the deep X-ray imaging survey of the Chandra Carina Complex Project (CCCP; see Townsley et al. 2011, for an overview), which mapped an area of about 1.4 square-degrees. With a detection limit corresponding to X-ray luminosities of about $10^{30} \mathrm{erg} / \mathrm{s}$, these X-ray data can detect the coronal X-ray emission of young stars down to $\sim 0.5 M_{\odot}$. The Chandra images revealed 14368 individual X-ray sources, and a sophisticated classification scheme showed that 10714 of these are most likely young stars in the Carina Nebula (Broos et al. 2011b).

To characterize these X-ray selected young stars, we performed a very deep near-infrared (NIR) survey with HAWK-I at the ESO 8 m-VLT (Preibisch et al. 2011c) that covered the central $\sim 0.36$ square-degree region of the Carina Nebula, which is about $30 \%$ of the CCCP area. The combination of the CCCP $\mathrm{X}$-ray data with these NIR data provided new information about the properties of the stellar populations in the entire complex (Preibisch et al. 2011b) and the stellar clusters Tr 16 and Tr 15 (Wolk et al. 2011; Wang et al. 2011). Further information about the X-ray detected young stellar populations was obtained in another study based on Spitzer mid-infrared observations (Povich et al. 2011). However, all these studies of the young stellar populations were focused on the central parts of the Carina Nebula, covering at most the 1.4 square-degree area observed in the CCCP; the outer parts of the extended cloud complex, which we denote as the Carina Nebula complex (CNC) in the following text, remained largely unexplored.

We have recently used the Herschel Space Observatory to produce very wide-field ( $\sim 6$ square-degrees) far-infrared (FIR) maps that cover the full spatial extent of the CNC in five bands between $70 \mu \mathrm{m}$ and $500 \mu \mathrm{m}$. These Herschel maps have revealed the morphology of the fainter cloud structures in the complex in unprecedented detail (Preibisch et al. 2012), allowed us to study the temperature and column-density structure of the clouds (Roccatagliata et al. 2013), and led to the detection of about 650 pre- and protostellar objects throughout the region (Gaczkowski et al. 2013). The cloud complex has an angular extension of $\sim 2.5^{\circ}$, which corresponds to physical scales of about $100 \mathrm{pc}$ at the distance of $2.3 \mathrm{kpc}$ (see Smith 2006) and covers an area of about four square-degrees on the sky. This implies that the associated population of young stellar objects also extends considerably beyond the currently well-studied central region of $\$ 1.4$ square-degrees.

NIR data are of fundamental importance for a reliable characterization of the stellar and circumstellar properties of the $\mathrm{X}$-ray and Herschel-detected objects. The lack of deep NIR data outside the $\approx 0.36$ square-degree HAWK-I field seriously limited all studies of the full stellar population of the CNC. For example, for most (78\%) of the 6896 X-ray sources outside of the HAWKI area, no NIR photometry is available, preventing a characterization of these young star candidates. Fundamental questions, such as about the spatial distribution of the young stars outside the central region of the Carina Nebula, or the full size of the young stellar population, could thus not be reliably addressed so far.

Clearly, a deep NIR survey that covers a much wider area than the previous HAWK-I survey, ideally the full $\sim 6$ squaredegree area that was studied with Herschel and Spitzer, is highly desirable. The combination of such NIR data with the X-ray and
Herschel source lists can reveal the full young stellar (and protostellar) populations across the entire Carina region, from the youngest deeply embedded protostars to $\sim 10$ Myr old T Tauri stars, for the first time. This will provide crucial constraints to the stellar mass functions and ages of different parts at the periphery of the complex and will yield new insight into the star formation history.

These arguments motivated our new wide-field NIR survey of the CNC with the Visible and Infrared Survey Telescope for Astronomy (VISTA), which is described in this paper. We denote this survey as the VISTA Carina Nebula Survey (VCNS). It covers $\mathrm{a} \approx 6.7$ square-degree area of the sky (see Fig. 1), which is 18.5 times larger in area than our previous HAWK-I survey. It represents the first deep NIR survey that covers the entire extent of the CNC. Although the VCNS is about two magnitudes less deep than the previous HAWK-I data, the photometric completeness limits are still about four magnitudes deeper than the nominal 2MASS completeness limits for crowded locations near the Galactic plane (see Skrutskie et al. 2006). As described below in more detail, the VCNS is sensitive enough to detect all young ( $\$ 5$ Myr old) stars, down to stellar masses of $0.1 M_{\odot}$, through extinctions of $A_{V} \approx 6 \mathrm{mag}$. It will thus serve as the basis for a comprehensive investigation of the full young stellar population in the entire CNC.

In Sect. 2 we provide a general description of our VISTA observations, the data reduction, and the creation and photometric calibration of the VCNS source catalog. Section 3 contains the characterization of the source catalog, which contains nearly four million individual objects and is available at the CDS. Section 4 describes the color-magnitude-diagram (CMD) for the entire region. In Sect. 5 we show VISTA images of selected regions in the $\mathrm{CNC}$ to demonstrate the image quality and continue with a "butterfly collection" of VISTA images of particularly interesting groups and clusters of young stellar objects (YSOs) in the outer parts of the Carina Nebula, together with a brief discussion of these objects. In the appendix, we also report on stars with high proper motions that were detected in our comparison of the VISTA images with the 2MASS data.

\section{VCNS observations and data analysis}

\subsection{VISTA observations}

We used the $4 \mathrm{~m}$ Visible and Infrared Survey Telescope for Astronomy (VISTA; Emerson et al. 2006) under ESO program number 088.C-0117(A) to obtain a deep $2 \times 2$ tile image mosaic covering a total sky area of $\approx 6.7$ square-degrees $\left(\approx 2.3^{\circ} \times 2.9^{\circ}\right)$ around the Carina Nebula in the NIR $J_{-}, H_{-}$, and $K_{\mathrm{s}}$-bands. The observed area is shown in Fig. 1; it includes not only the entire CCCP field, but also almost the full area that was studied in our Herschel survey. We denote the four individual mosaic field positions as NE (centered on J2000 celestial coordinates RA $\approx$ $10^{\mathrm{h}} 47^{\mathrm{m}} 00^{\mathrm{s}}$, Dec $\left.\approx-58^{\circ} 37^{\prime} 40^{\prime \prime}\right), \mathrm{NW}\left(\mathrm{RA} \approx 10^{\mathrm{h}} 38^{\mathrm{m}} 43^{\mathrm{s}}\right.$, Dec $\approx$ $\left.-58^{\circ} 37^{\prime} 35^{\prime \prime}\right), \mathrm{SW}\left(\mathrm{RA} \approx 10^{\mathrm{h}} 38^{\mathrm{m}} 33^{\mathrm{s}}\right.$, Dec $\left.\approx-60^{\circ} 04^{\prime} 50^{\prime \prime}\right)$, and $\mathrm{SE}\left(\mathrm{RA} \approx 10^{\mathrm{h}} 47^{\mathrm{m}} 15^{\mathrm{s}}\right.$, Dec $\left.\approx-60^{\circ} 04^{\prime} 50^{\prime \prime}\right)$. The VISTA observations were performed in March 2012 under good photometric conditions and subarcsecond (near-infrared) seeing conditions (see Table 1).

The VISTA infrared camera VIRCAM (see Dalton et al. 2006) is composed of an array of sixteen individual $2048 \times$ 2048 pixel Raytheon VIRGO HgCdTe infrared detectors that cover a field of view of 1.65 degree diameters with a nominal mean pixel size of $0.34^{\prime \prime}$. As a result of the gaps between the individual infrared detectors, VIRCAM produces sparsely 


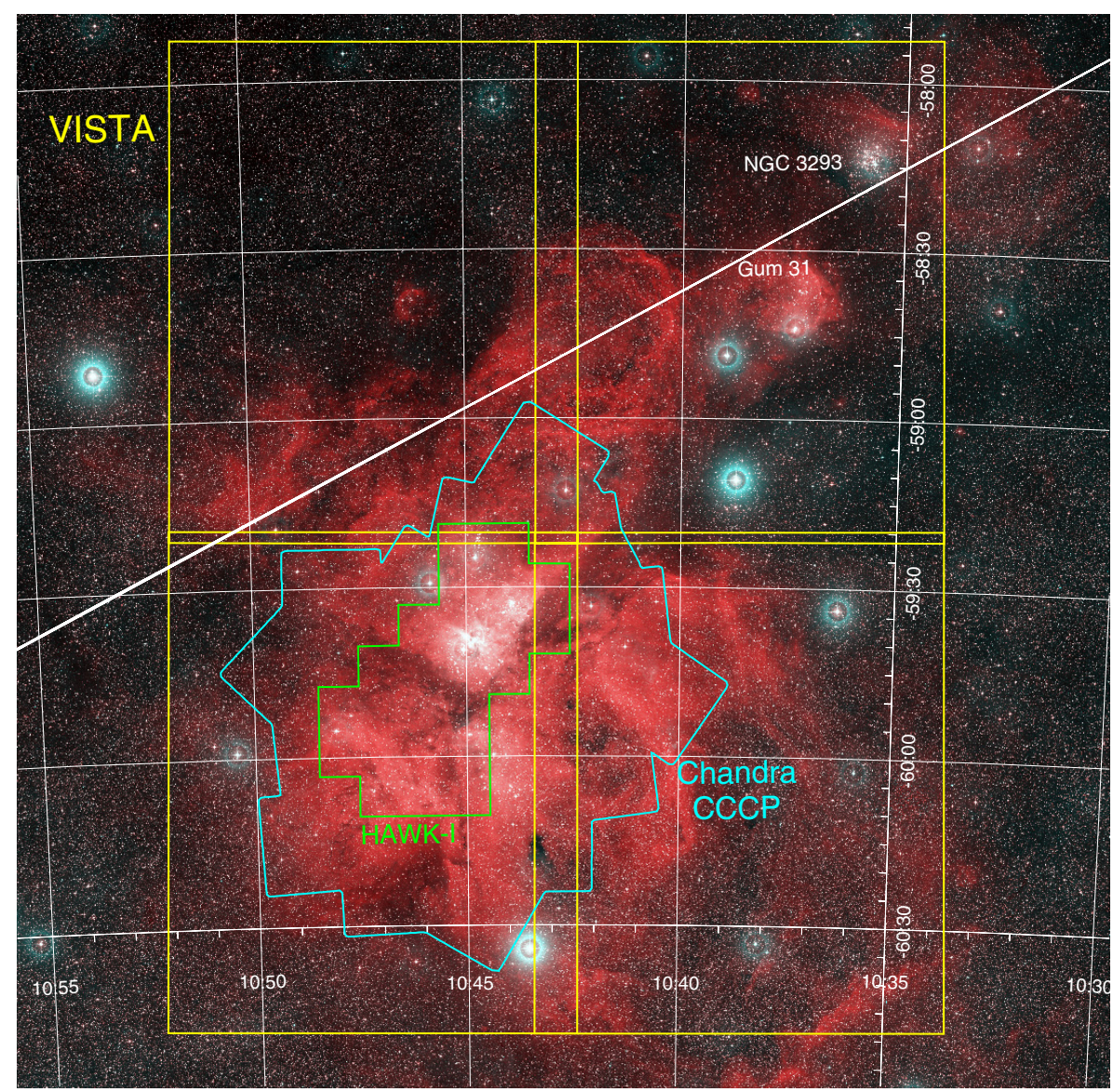

Fig. 1. Outline of the VISTA Carina Nebula Survey mosaic pattern (yellow boxes) shown in the optical image of the Carina Nebula complex (from: www . eso.org/public/images/eso0905b/; image credit: ESO/Digitized Sky Survey 2, Davide De Martin). A grid of J2000 coordinates is shown. The famous star $\eta \mathrm{Car}$ in the center of the Carina Nebula is located slightly to the left and below the image center (near $\mathrm{RA}(\mathrm{J} 2000)=10^{\mathrm{h}} 45^{\mathrm{m}}$, $\left.\operatorname{Dec}(\mathrm{J} 2000)=-59^{\circ} 41^{\prime}\right)$. The H II region Gum 31 at the northwestern edge of the bright nebulosity and the stellar cluster NGC 3293 near the northwestern edge of the VISTA field are labeled. The region observed in the context of the Chandra Carina Complex Project (CCCP; Townsley et al. 2011) and the area of the HAWK-I survey (Preibisch et al. 2011c) are marked. The Galactic plane is shown as the thick solid line running approximately diagonal through the northern part of the VCNS area.

Table 1. Observing parameters for the VISTA tiles used for the VCNS.

\begin{tabular}{|c|c|c|c|c|c|c|c|}
\hline Start UT date and time & $\overline{\text { Tile/ }}$ & and & $\begin{array}{c}\text { Seeing } \\
{[\operatorname{arcsec}]}\end{array}$ & Airmass & $\begin{array}{c}\text { Observing time } \\
\text { NJIT } \times \text { NDIT } \times \text { DIT }\end{array}$ & $\begin{array}{r}5 \sigma-\text { limit } \\
{[\mathrm{mag}]}\end{array}$ & $\begin{array}{r}\text { Number of } \\
\text { sources }\end{array}$ \\
\hline $2012-03-0705: 30: 06.077$ & $\mathrm{NE}$ & $J$ & 0.95 & $1.232-1.233$ & $5 \times 15 \times 5 \mathrm{~s}$ & 20.4 & 1001102 \\
\hline 2012-03-24 01:27:18.088 & $\mathrm{NE}$ & $H$ & 0.90 & $1.294-1.291$ & $5 \times 27 \times 2 s$ & 19.4 & 1062123 \\
\hline 2012-03-19 01:12:10.321 & $\mathrm{NE}$ & $K_{\mathrm{s}}$ & 0.85 & $1.362-1.359$ & $5 \times 20 \times 2 s$ & 18.7 & 1082032 \\
\hline 2012-03-06 03:56:13.501 & NW & $J$ & 0.93 & $1.210-1.209$ & $5 \times 15 \times 5 s$ & 20.4 & 910286 \\
\hline 2012-03-08 06:29:51.344 & NW & $H$ & 0.85 & $1.334-1.337$ & $5 \times 27 \times 2 s$ & 19.4 & 1035387 \\
\hline 2012-03-06 02:36:52.775 & NW & $K_{\mathrm{s}}$ & 0.90 & $1.282-1.280$ & $5 \times 20 \times 2 s$ & 18.8 & 996310 \\
\hline 2012-03-07 03:33:20.232 & SW & $J$ & 0.89 & $1.240-1.239$ & $5 \times 15 \times 5 \mathrm{~s}$ & 19.9 & 1122918 \\
\hline 2012-03-23 05:42:56.679 & SW & $H$ & 0.83 & $1.380-1.384$ & $5 \times 27 \times 2 s$ & 19.4 & 1255008 \\
\hline 2012-03-15 01:12:14.904 & SW & $K_{\mathrm{s}}$ & 1.01 & $1.399-1.395$ & $5 \times 20 \times 2 s$ & 18.5 & 956150 \\
\hline 2012-03-07 02:49:12.235 & SE & $J$ & 0.96 & $1.292-1.290$ & $5 \times 10 \times 5 s$ & 20.1 & 948204 \\
\hline 2012-03-09 04:46:02.438 & SE & $H$ & 0.84 & $1.231-1.232$ & $5 \times 27 \times 2 s$ & 19.4 & 1261207 \\
\hline 2012-03-11 04:49:23.415 & SE & $K_{\mathrm{s}}$ & 0.97 & $1.236-1.237$ & $5 \times 20 \times 2 s$ & 18.5 & 978652 \\
\hline
\end{tabular}

Notes. The first column lists the UT date and time of the start of the first pawprint observation of each tile. The listed seeing values represent the average FWHM of stellar point spread functions as measured on the images. The final column lists the number of point-like objects that were identified in the CASU processing of each tile.

sampled images of the sky that are known as pawprints. To obtain an image without gaps and with (almost) uniform sky coverage, six individual pawprints with specific offsets in $x$ - and $y$-direction are combined to yield a so-called tile. The observing procedure was as follows: Individual images were obtained as the sum of NDIT exposures with detector integration time DITs, and this sequence was repeated at five different jitter positions (NJIT) with jitter offsets of $15^{\prime \prime}$. The whole procedure was performed for six different pawprint offset positions to provide enough data for a completely filled tile. Details are given in Table 1.

\subsection{Data reduction and image creation}

The raw data were reduced and the individual pawprints were combined to the final tiles at the Cambridge Astronomy Survey Unit (CASU) with the VISTA data flow system (VDFS; CASU CIRDR Version 1.182; see Irwin et al. 2004). Details of these processing steps are described on the CASU webpage ${ }^{1}$. The analysis adjusts the sky level on all component images to the

\footnotetext{
1 http://apm49.ast.cam.ac.uk/surveys-projects/vista/ technical/
} 
same level, projects the individual detector images onto a single tangent plane World Coordinate System image, and corrects for the photometric distortion within the pawprints that results from the geometric distortion in the VISTA wide-field images.

The images were astrometrically calibrated (also as a part of the CASU processing) by using about 4000 2MASS stars per tile to derive an World Coordinate System fit. The relative accuracy of the astrometry is between 60 and 70 mas (rms).

\subsection{Source detection and flux extraction}

The VDFS at CASU also performed the source detection and flux extraction in each tile. The number of detected sources in each tile is listed in Table 1. Aperture photometry for each of these sources was performed (as part of the CASU processing steps) by sky-corrected integration over a series of soft-edged circular apertures with different radii. Furthermore, the VDFS pipeline determined aperture corrections with a curve-of-growth analysis from a number of selected sources in each tile. For our photometric catalog described below we used the fluxes based on the extraction and corresponding correction for aperture size 3 (as recommended by CASU for wide-field surveys), for which the radius is set to $1^{\prime \prime}$. The corresponding aperture corrections range between 0.2 and $0.3 \mathrm{mag}$, depending on the band and the seeing conditions during the observation for the individual tiles.

The results of this reduction are stored in source catalogs for each individual tile. The structure of these catalogs is described at the CASU webpage ${ }^{2}$. The formal $5 \sigma$ magnitude limit for point-like sources listed in Table 1 was calculated as specified by CASU from the measured sky noise in each tile.

As part of the standard CASU pipeline analysis, zero points are determined for each tile catalog, following the strategies described in Hodgkin et al. (2009) and at the CASU webpage ${ }^{3}$. The scatter of all zero-point measurements in any particular passband for each specific night indicates the photometric quality of this night. For all the tiles used for our study, this scatter in the nightly zero points is $\leq 0.022 \mathrm{mag}$ in the $J$-band, $\leq 0.015 \mathrm{mag}$ in the $H$-band, and $\leq 0.011 \mathrm{mag}$ in the $K_{\mathrm{s}}$-band. This shows that all our tiles were obtained under (very) good photometric conditions.

\subsection{Photometric calibration}

The photometric calibration of the tile catalogs was obtained from 2MASS stars that are present in large numbers (several thousands) in each tile (i.e., are observed simultaneously with the target stars). The source fluxes (in units of counts per second) listed in the CASU catalogs refer to radiation fluxes in the VISTA photometric system, which is the natural detector+filter system based on Vega. The VISTA system is tied to and similar, but not identical, to the 2MASS system. Therefore, color-terms are required for a transformation between the VISTA and the 2MASS system.

According to the detector integration times used and the seeing conditions during our observations, objects brighter than $J \approx 11.8, H \approx 11.25$, or $K_{\mathrm{s}} \approx 10.5$ are expected to be in the nonlinear or saturated regime of the VIRCAM detectors. These limits were verified in our comparison of VISTA and 2MASS magnitudes described in Sect. 3.1. to exclude such saturation

\footnotetext{
2 http://apm49.ast.cam.ac.uk/surveys-projects/vista/ technical/catalogue-generation

3 http://apm49.ast.cam.ac.uk/surveys-projects/vista/ technical
}

or nonlinearity effects from our final photometry catalog, we replaced the VISTA photometry for all sources brighter than $J \approx 12.0, H \approx 11.75$, or $K_{\mathrm{s}} \approx 11.5$ by photometry from the 2MASS point source catalog. This affects many of the bright stars in our survey field, including most of the young O- and early B-type members of the Carina Nebula (see Smith 2006, for a list). To obtain a homogeneous and consistent photometric catalog, we decided to calibrate the instrumental VISTA magnitudes to the 2MASS system. The steps in this calibration are described below.

\subsubsection{Band merging}

The band-merged catalogs for each tile are based on the source positions in the $H$-band because this filter band shows the largest number of source detections. For each $H$-band source, we searched for counterparts in the $J$-band and $K_{\mathrm{s}}$-band catalogs; counterpart candidates with spatial offsets of less than $0.3^{\prime \prime}$ were considered to be matches.

\subsubsection{Identification of 2 MASS calibrators}

To construct a sample of calibrators, we started with the $\approx 50000$ 2MASS point source catalog objects per tile with photometric quality AAA that can be identified with a VISTA source by a positional offset criterion of $\leq 0.5^{\prime \prime}$. From these, we then selected 2MASS sources in the magnitude ranges $12.0 \leq K_{\mathrm{s}} \leq 13.5$ (as recommended in the VISTA/VIRCAM manual) and $J \geq 12.0$ and $H \geq 12.0$. These stars are (i) faint enough not to be in the nonlinear or saturated regime of the VISTA data, and, at the same time; (ii) bright enough to have high-quality photometric measurements. This selection process led to 17131 2MASS calibration sources in the NW tile, 17499 in the NE tile, 20175 in the SW tile, and 20675 in the SE tile.

\subsubsection{Calibrating fluxes to magnitudes}

The calibration procedure started with extinction-corrected instrumental magnitudes, which were computed from the aperture fluxes given in the VIRCAM catalogs, the appropriate aperture corrections, and the mean atmospheric extinction during the observation (as stored in the CASU Headers of the tile files).

The calibration to the 2MASS system was made by solving linear equations relating these extinction-corrected instrumental magnitudes and colors to the known 2MASS magnitudes and colors for the several thousand 2MASS calibrators in each tile. The inversion of these equations then allowed computing calibrated magnitudes in the 2MASS system from the extinctioncorrected instrumental magnitudes of all stars.

Finally, we also compared our calibration with the recent study of Soto et al. (2013), who presented the color transformation of the 136 million stars from the VISTA Variable in the Via Lacerta survey into the 2MASS system. This showed a very good agreement between the color terms derived in our calibration and those determined by Soto et al. (2013).

\subsection{Creation of the final combined source catalog}

The combined VCNS source catalog was finally constructed by merging all individual tile catalogs. Duplicates from the overlap regions in our $2 \times 2$ tile mosaic were identified with a spatial offset criterion of $\leq 0.5^{\prime \prime}$, and only the catalog entry with the higher signal-to-noise ratio $(\mathrm{S} / \mathrm{N})$ was included in the merged catalog. 
Table 2. VCNS near-infrared photometry catalog.

\begin{tabular}{|c|c|c|c|c|c|c|c|}
\hline $\begin{array}{c}\text { VCNS - J } \\
\text { [ J2000 ] } \\
(1)\end{array}$ & $\begin{array}{r}J \\
{[\mathrm{mag}]} \\
(2)\end{array}$ & $\begin{array}{r}H \\
{[\mathrm{mag}]} \\
(3) \\
\end{array}$ & $\begin{array}{r}K_{\mathrm{s}} \\
{[\mathrm{mag}]} \\
(4)\end{array}$ & $\begin{array}{r}S / N \\
\left(F_{J}\right) \\
(5) \\
\end{array}$ & $\begin{array}{r}S / N \\
\left(F_{H}\right) \\
(6) \\
\end{array}$ & $\begin{array}{r}S / N \\
\left(F_{K \mathrm{~s}}\right) \\
(7) \\
\end{array}$ & $\begin{array}{c}\text { PHOT } \\
{[0,1,2]} \\
(8)\end{array}$ \\
\hline $103333.40-604729.5$ & 16.78 & 16.27 & 16.14 & 76.3 & 60.9 & 34.6 & 0 \\
\hline $103333.42-604722.7$ & 15.83 & 15.22 & 15.01 & 140.2 & 121.7 & 78.8 & 0 \\
\hline $103333.43-604717.0$ & 14.37 & 13.61 & 13.45 & 311.6 & 295.9 & 200.1 & 0 \\
\hline $103333.49-604637.3$ & 18.63 & 17.09 & 16.94 & 19.2 & 32.3 & 17.8 & 0 \\
\hline $103333.50-604655.8$ & 15.41 & 14.97 & 14.90 & 177.7 & 141.9 & 84.7 & 0 \\
\hline $103333.57-604713.7$ & 18.11 & 17.54 & 17.30 & 28.0 & 22.5 & 13.2 & 0 \\
\hline $103333.62-604557.4$ & -9.99 & 17.47 & 15.89 & -9.9 & 21.6 & 39.0 & 0 \\
\hline 103333.64-604643.5 & 17.44 & 16.95 & 16.90 & 47.4 & 36.3 & 18.6 & 0 \\
\hline $103333.67-604653.6$ & 17.33 & 16.73 & 16.60 & 51.6 & 43.2 & 23.9 & 0 \\
\hline$\ldots$ & $\cdots$ & & $\cdots$ & $\cdots$ & & $\cdots$ & \\
\hline $105216.92-604657.7$ & 19.26 & 18.48 & -9.99 & 9.2 & 10.5 & -9.9 & 0 \\
\hline
\end{tabular}

Notes. The full Table 2 is only available at the CDS. A portion is shown here for guidance regarding its form and content. The meaning of the columns is as follows: Col. (1): Source name, based on the J2000 celestial coordinates. Columns (2) to (4): Near-infrared photometry in the $J-, H-$, and $K_{\mathrm{s}}$-bands. Objects without photometry in a band are marked by the number -9.99 . Columns (5) to (7): Signal-to-noise ratio of the individual photometric flux measurements in Cols. (2) to (4). Column (8): Flag indicating the source of the photometry: $0=$ photometry from VISTA data, $1=$ photometry from 2 MASS catalog, 2 = no photometry available.

The final catalog contains data on those 3951580 VISTA sources that were detected in at least two of the three bands. This catalog is published as Table available at the CDS; a small part is shown in Table 2. There are two reasons why we did not include single-band detections: first, a photometric calibration with color terms is only possible for sources with at least two band detections. Second, the requirement of a detection in two different bands (i.e., independent data sets) efficiently excludes possible spurious objects and thus increases the reliability of the final catalog.

\section{Characterization of the VCNS catalog}

The final VCNS catalog contains 3951580 individual NIR sources that are detected in at least two bands; 3191676 of these (i.e., $\approx 81 \%$ ) are detected in all three bands.

As mentioned above, VISTA photometry was not used for bright sources that exceeded at least one of the magnitude limits $J<12.0, H<11.75$, or $K_{\mathrm{s}}<11.5$. This concerns 24552 bright stars. For 24086 of these, a clear and unique 2MASS counterpart could be identified and the photometry reported in our catalog was then taken from 2 MASS. The remaining bright objects were problematic cases, for example, because a 2MASS source is resolved into several counterparts in the VISTA images. Sometimes, very bright stars have no entry in the 2MASS point source catalog, although they are clearly visible as apparently single point-like source in the 2MASS images. This effect is often related to the presence of a nearby very bright star, which apparently caused detection problems in the 2MASS catalog in a similar way as we discuss below for the VCNS catalog. Although no photometry can be given for these cases, we kept these objects in the catalog for the sake of completeness, but flagged them accordingly in the catalog.

Many of the very bright stars (magnitudes $\$ 7$ ) are so heavily saturated in the VISTA images that they were not recognized as point-like sources by the detection routine; these objects are therefore missing from the catalog.

\subsection{Photometric quality}

The total uncertainties of the photometry consist of two components: the uncertainties associated with the flux measurements of each individual source, and the uncertainties related to the calibration of the fluxes with respect to the magnitudes in the 2MASS system.

The uncertainties of the individual flux measurements from the VISTA tiles are provided by CASU and listed as the signalto-noise ratio, that is, the inverse of the relative flux errors $(\Delta F / F)$, in our catalog. The corresponding uncertainties in magnitude units are shown in Fig. 2. The relative flux errors are lower than $10 \%$ for most objects brighter than $J \approx 19 \mathrm{mag}$, $H \approx 18.5 \mathrm{mag}$, and $K_{\mathrm{s}} \approx 17.5 \mathrm{mag}$.

To quantify the quality of the photometric calibration of our final catalog, we performed a comprehensive comparison of the VISTA magnitudes with all suitable 2MASS stars in the tiles. A comparison sample was constructed from all those 2MASS stars that can be identified within $\leq 0.5^{\prime \prime}$ with a VISTA cata$\log$ object, for which no other object is found within $3.5^{\prime \prime}$ (to avoid possible contamination of the photometry), and which have flux measurements with a $\mathrm{S} / \mathrm{N}$ of at least 10 in all three filter bands. This sample consists of 137230 objects. The standard deviations ${ }^{4}$ of the VISTA versus 2 MASS magnitudes and colors are $\sigma(J)=0.050 \mathrm{mag}, \sigma(H)=0.049 \mathrm{mag}, \sigma\left(K_{\mathrm{s}}\right)=0.061 \mathrm{mag}$, $\sigma(J-H)=0.056 \mathrm{mag}$, and $\sigma\left(H-K_{\mathrm{s}}\right)=0.066 \mathrm{mag}$.

\subsection{Detection efficiency}

Our detailed visual inspection of the VISTA images showed that in some specific locations, some point-like objects, which are clearly visible in the images, are missing from the CASU catalogs. The occurrence of this problem is often related to the presence of very bright stars. The exact reason for this problem in

4 To exclude the effects of outliers (e.g., due to possible mismatches between 2MASS and VISTA objects) we report here a robust estimate of the standard deviation based on the normalized median absolute deviation. This is a robust measure of the variability in a data set that is scaled to be approximately equal to the standard deviation for normally distributed data (see, e.g., Feigelson \& Babu 2012). 

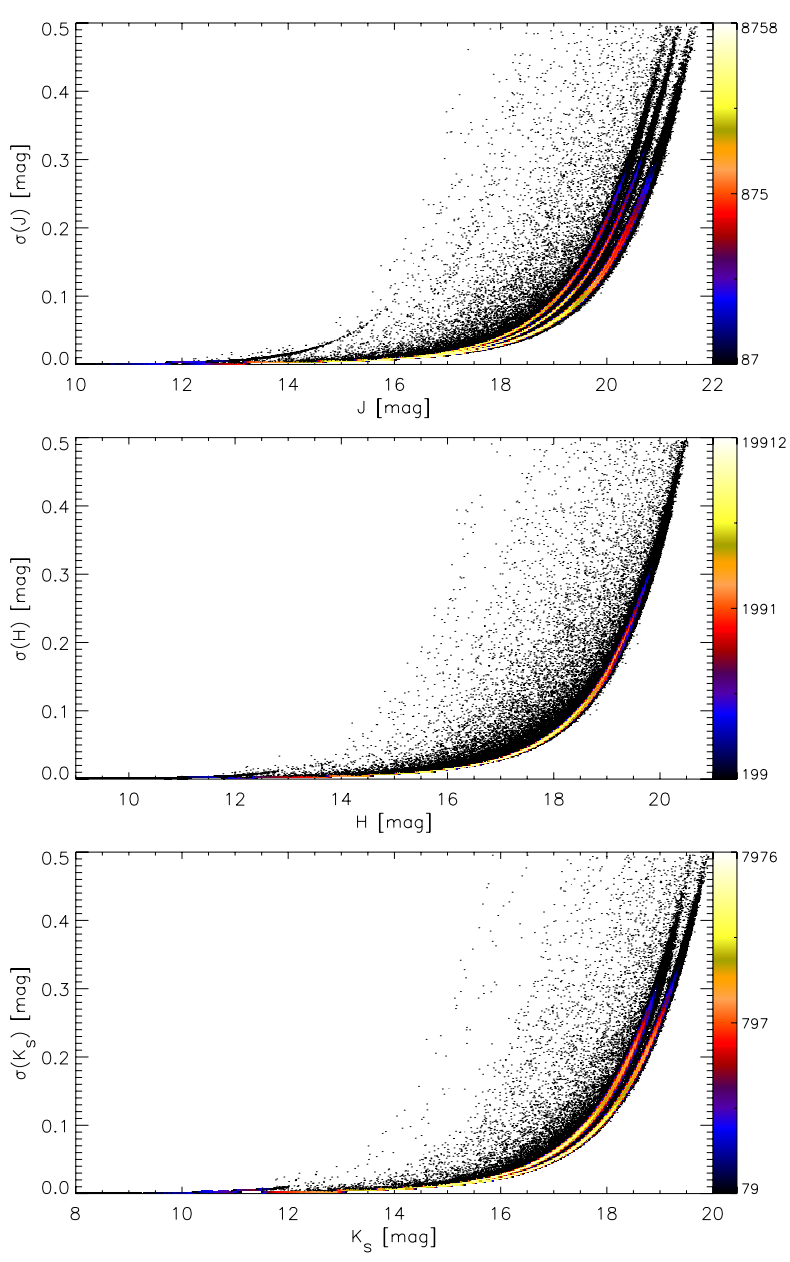

Fig. 2. Hess diagrams for the distribution of the uncertainties in the source magnitudes as a function of the source magnitude for the $J$-band (top), the $H$-band (middle), and the $K_{\mathrm{s}}$-band (bottom). The color scale shows the number of objects in $2 \mathrm{D}$ bins with a size of $0.025 \mathrm{mag}$ on the $x$-axis and 0.002 mag on the $y$-axis. In all bins with fewer objects than the lower limit indicated at the bottom of the color bar, the individual objects are plotted by black dots.

the automatic source detection routine could not be established. While this leads to a low degree of incompleteness in the catalog, our analysis showed that the total area affected by this problem is very small, just about $0.5 \%$ of our total survey area. We note that the 2MASS point source catalog suffers from a similar effect of incompleteness near particularly bright sources.

Another problem can result from crowding in regions with a very high source density. This effect is, however, mainly restricted to the extremely dense cluster $\operatorname{Tr} 14$ near the center of the Carina Nebula, where the very strong source crowding leads to considerable incompleteness of the VISTA catalog. Observations with much better angular resolution are required for detailed studies of such dense clusters; for $\operatorname{Tr} 14$, we note that results of adaptive-optics observations have been presented by Ascenso et al. (2007) and Sana et al. (2010).

\subsection{Detection and completeness limits}

The formal $5 \sigma$ magnitude limits for point-like sources in the VCNS are $J_{5 \sigma} \approx 20.0, H_{5 \sigma} \approx 19.4$, and $K_{\mathrm{s}, 5 \sigma} \approx 18.5$, but numerous objects with fainter magnitudes are also reliably detected in at least two bands and listed in the catalog. The faintest

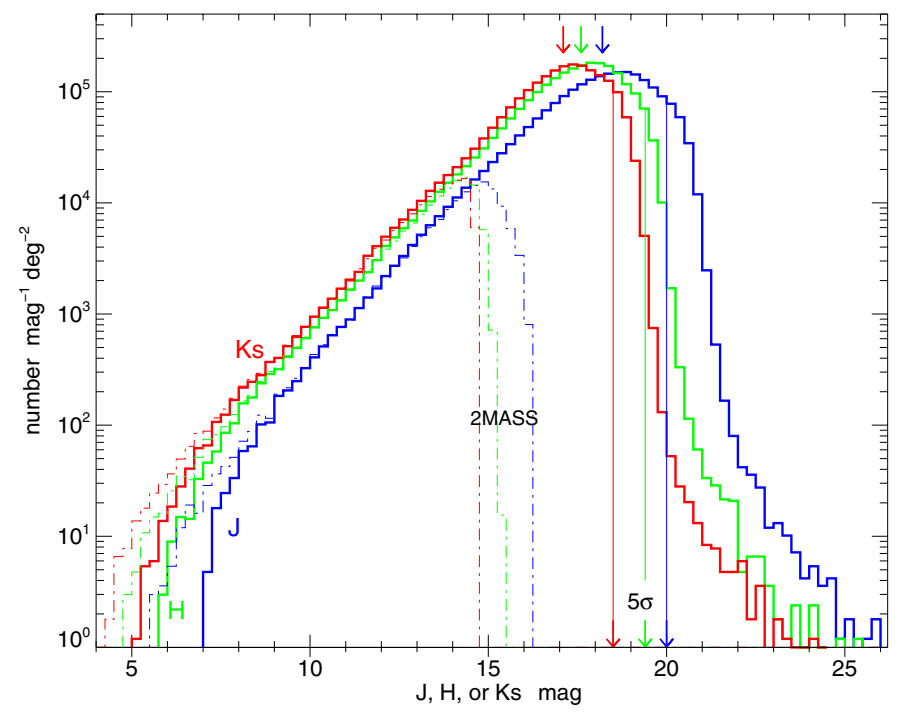

Fig. 3. Histogram of the NIR magnitudes of the objects in the VCNS catalog (solid lines). The blue, green, and red histograms refer to the $J$-, $H$-, and $K_{\mathrm{s}}$-band, respectively. The dashed lines show the corresponding distributions for the 2MASS point source catalog sources in the same area. The downward pointing arrows below the upper $x$-axis mark our estimates of the typical completeness limits of the VISTA data, while the vertical lines with arrows at the lower $x$-axis mark the $5 \sigma$ magnitude limits.

$S / N \geq 3$ detections in the $J$-band have $J \approx 21.2$ mag, the faintest $S / N \geq 3$ detections in the $H$-band have $H \approx 19.9 \mathrm{mag}$, and the faintest $S / N \geq 3$ detections in the $K_{\mathrm{s}}$-band have $K_{\mathrm{s}} \approx 19.3 \mathrm{mag}$.

According to the pre-main sequence stellar models of Siess et al. (2000), $6 \mathrm{Myr}$ old $^{5}$ stars of mass $M=0.1 M_{\odot}$ at a distance of $2.3 \mathrm{kpc}$ have expected NIR magnitudes of $J \approx 18.4$, $H \approx 17.8$, and $K_{\mathrm{s}} \approx 17.4$. This shows that $0.1 M_{\odot}$ stars with extinctions up to $A_{J} \approx 1.6 \mathrm{mag}$ (corresponding approximately to $A_{V} \approx 5.7 \mathrm{mag}$ ) are above the $5 \sigma$ magnitude limits of the VCNS data.

A reliable determination of a unique completeness limit that would be appropriate for the full wide-field survey is not feasible. The main reason for this is the strong and highly variable diffuse emission that pervades large parts of the CNC. At positions with bright nebulous emission, the source detection sensitivity can be locally degraded. Instead, we derived an empiric estimate for the typical completeness limit from the magnitude distributions of the VCNS catalog objects as shown in Fig. 3. The points in the magnitude histograms where the distributions start to depart significantly from the power-law increase observed at bright magnitudes can serve as a proxy for the typical completeness limit across the field. In this way, we derived limits of $J_{\text {compl }} \sim 18.2 \mathrm{mag}, H_{\text {compl }} \sim 17.5 \mathrm{mag}$, and $K_{\text {s, compl }} \sim 17.1 \mathrm{mag}$. The numbers can be viewed as the limits above which almost all objects are reliably detected in the full survey area. We note that nearly all objects above these limits are detections with a $S / N \geq 10$. We note that in regions with strong nebulosity (especially in the central parts of the Carina Nebula), the actual completeness limit may be at somewhat brighter magnitudes, whereas in the outer parts of our survey area the completeness is probably somewhat better than the quoted values. In conclusion, we can expect that outside the brightest parts of

5 This value is an approximate upper limit for the ages of most stellar clusters in the Carina Nebula (e.g., Dias et al. 2002; Preibisch et al. 2011c). 
the central nebula, almost all young stars (with ages of up to $\sim 6 \mathrm{Myr}$ ) in the CNC with extinctions of up to $A_{V} \approx 5$ mag are included in the VCNS source catalog.

\section{Stellar populations in the VCNS field}

From comparing the number of almost four million VCNS catalog objects with the estimates of the total young stellar population of the Carina Nebula (an estimate of $\sim 100000$ young stars in the 1.4 square-degree area of the CCCP was derived by Feigelson et al. 2011), it is obvious that the vast majority of all catalog objects must be field stars. The high degree of background contamination is a consequence of the location of the Carina Nebula very close to the Galactic plane (which runs roughly diagonally through the northern parts of the VCNS survey area; see Fig. 1). Considering the $5 \sigma$ detection limits of the VCNS catalog and the absolute NIR magnitudes of $1 M_{\odot}$ main-sequence stars, we find that Sun-like field stars can be detected up to distances of $11.1 \mathrm{kpc}$ in the $K_{\mathrm{s}}$-band, and $18.7 \mathrm{kpc}$ in the $J$-band (neglecting extinction). At the Galactic longitude of the Carina Nebula $\left(l \approx-72^{\circ}\right)$, this implies that we sample almost the entire Galactic disk stellar population with $M \gtrsim 1 M_{\odot}$ along this line of sight. The VISTA catalog is thus clearly dominated by (mostly background) field stars, and the identification of young stars associated with the CNC is not straightforward without additional information.

Figure 4 shows the CMD for all sources in the VCNS cata$\log$. In the upper part of the CMD, for $J \lesssim 12$, the two stellar populations of field dwarf stars (with typical colors in the range $J-H \approx[0-0.3])$ and giants $(J-H \approx[0.6-0.9])$ are clearly visible, as typically seen in CMDs based on wide-field NIR observations. This upper part of the CMD contains foreground field stars as well as numerous moderately distant background stars with low degrees of reddening.

At fainter magnitudes $(J \gtrsim 14)$, the peak of the observed $J-H$ color distribution shifts toward the range [0.6-1.0], which is typical for moderately reddened low-mass background field stars.

The position of the background field stars in the CMD is affected by the reddening of the clouds associated with the $\mathrm{CNC}$. Based on the cloud column density maps derived from the Herschel observations of the CNC (see Figs. 6 and 7 in Preibisch et al. 2012), the typical cloud extinction in the CNC is in the range of $A_{V} \approx[0.5-3]$ mag (corresponding to a reddening of $E(J-H) \approx[0.05-0.3])$; somewhat higher values up to $A_{V} \lesssim 10$ mag (corresponding to $E(J-H) \lesssim 1$ ) are seen in a few percent of the nebula area, while higher values are restricted to the area of a few very compact dense clumps.

The intermediate-mass pre-main sequence stars in the Carina Nebula are expected to appear in the faint extension of the background giant band for $J \geq 13$. For $J \geq 15$, the low-mass ( $\left.\lesssim 1 M_{\odot}\right)$ pre-main sequence stars in the Carina Nebula will appear in the red wing of the background field star bulge.

The red boundary of the distribution in the CMD confirms that the line-of-sight extinction is typically $A_{V} \leq 10 \mathrm{mag}$ and is predominantly caused by the $\mathrm{CNC}$ clouds.

\section{VISTA images of interesting objects}

\subsection{Near-infrared morphology of selected regions}

The full VISTA mosaic image is much too large for a complete and detailed display, therefore we present here images of just a few selected regions in the survey field that were not extensively

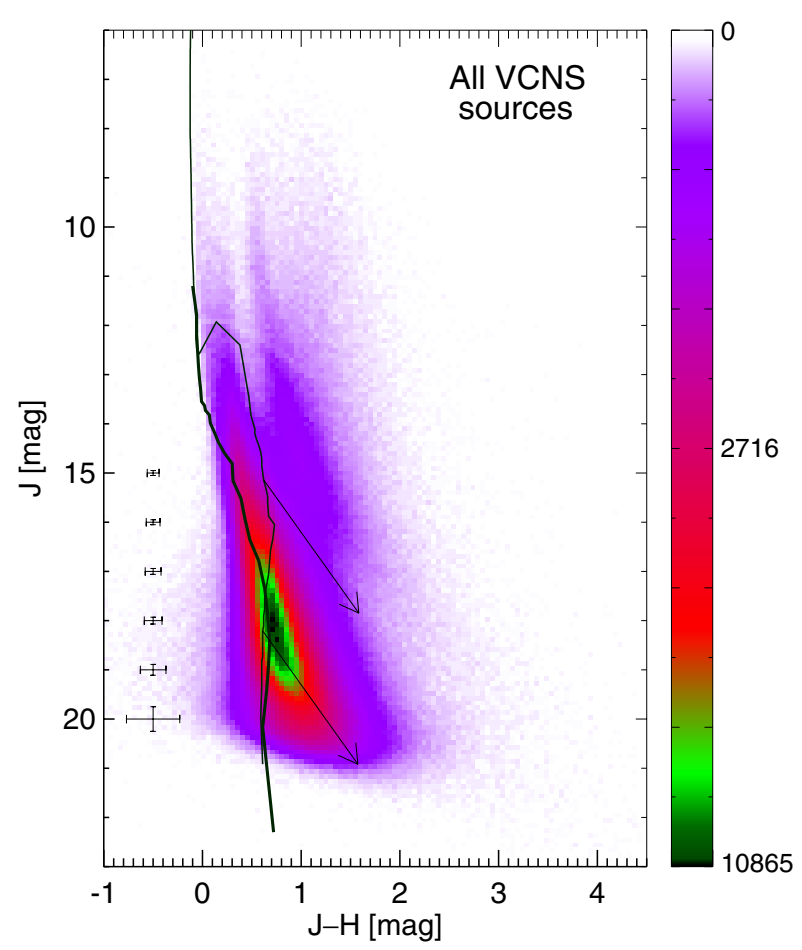

Fig. 4. Hess diagram of the color-magnitude distribution of all objects in the VCNS catalog. The color indicates the number of objects in each bin with a size of $0.05 \mathrm{mag}$ in $J-H$ and $0.1 \mathrm{mag}$ in $J$, using a squareroot intensity scale. The thick solid line shows the ZAMS from Siess et al. (2000) at the distance of the Carina Nebula $(2.3 \mathrm{kpc})$, while the thin line shows the $3 \mathrm{Myr}$ (the typical mean age of the young stellar population in the Carina Nebula; see Getman et al. 2014) isochrone composed from the models of Baraffe et al. (1998) for the mass range 0.02 to $0.5 M_{\odot}$, Siess et al. (2000) for the mass range 0.5 to $7 M_{\odot}$, and Lejeune \& Schaerer (2001) (model iso-c020-0650) for the mass range 7 to $70 M_{\odot}$. The arrows indicate reddening vectors for $A_{V}=10 \mathrm{mag}$ (assuming $A_{J}=0.271 A_{V}$ and $A_{H}=0.175 A_{V}$; Draine 2011) starting at the location of $3 \mathrm{Myr}$ old stars with masses of $1 M_{\odot}$, and $0.1 M_{\odot}$. The row of error bars in the left part of the plot shows the typical magnitudedependent photometric uncertainties.

observed so far. Near-infrared images of the central parts of the Carina Nebula are already available in the literature (see, e.g., the HAWK-I images presented in Preibisch et al. 2011c) and at various websites $^{6}$, so we focus here on objects at the periphery of the complex.

The location and size of all the VISTA images shown in this section is marked in Fig. 5 on top of the Herschel $160 \mu$ m map.

\subsubsection{Gum $31 \mathrm{HII}$ region}

Gum 31 (first described by Gum 1955) is an H II region at the northwestern edge of the Carina Nebula (see Fig. 1). It appears quite prominent in optical images ${ }^{7}$, and the small-scale morphology of the western part of the ionization front was impressively visualized by imaging with the Hubble Space Telescope ${ }^{8}$. The $\mathrm{H}$ II region is excited by the two O6.5V stars HD $92206 \mathrm{~A}$ and B and the O8.5V star HD 92206 C (Maíz-Apellániz et al. 2004) in the young stellar cluster NGC 3324, which appears close to the center of the VISTA image shown in Fig. 6. Note the numerous

\footnotetext{
6 See, e.g., http://www. eso.org/public/news/eso1208/

7 E.g., ESO Photo Release www . eso.org/public/news/eso1207/

8 hubblesite.org/newscenter/archive/releases/2008/34/
} 


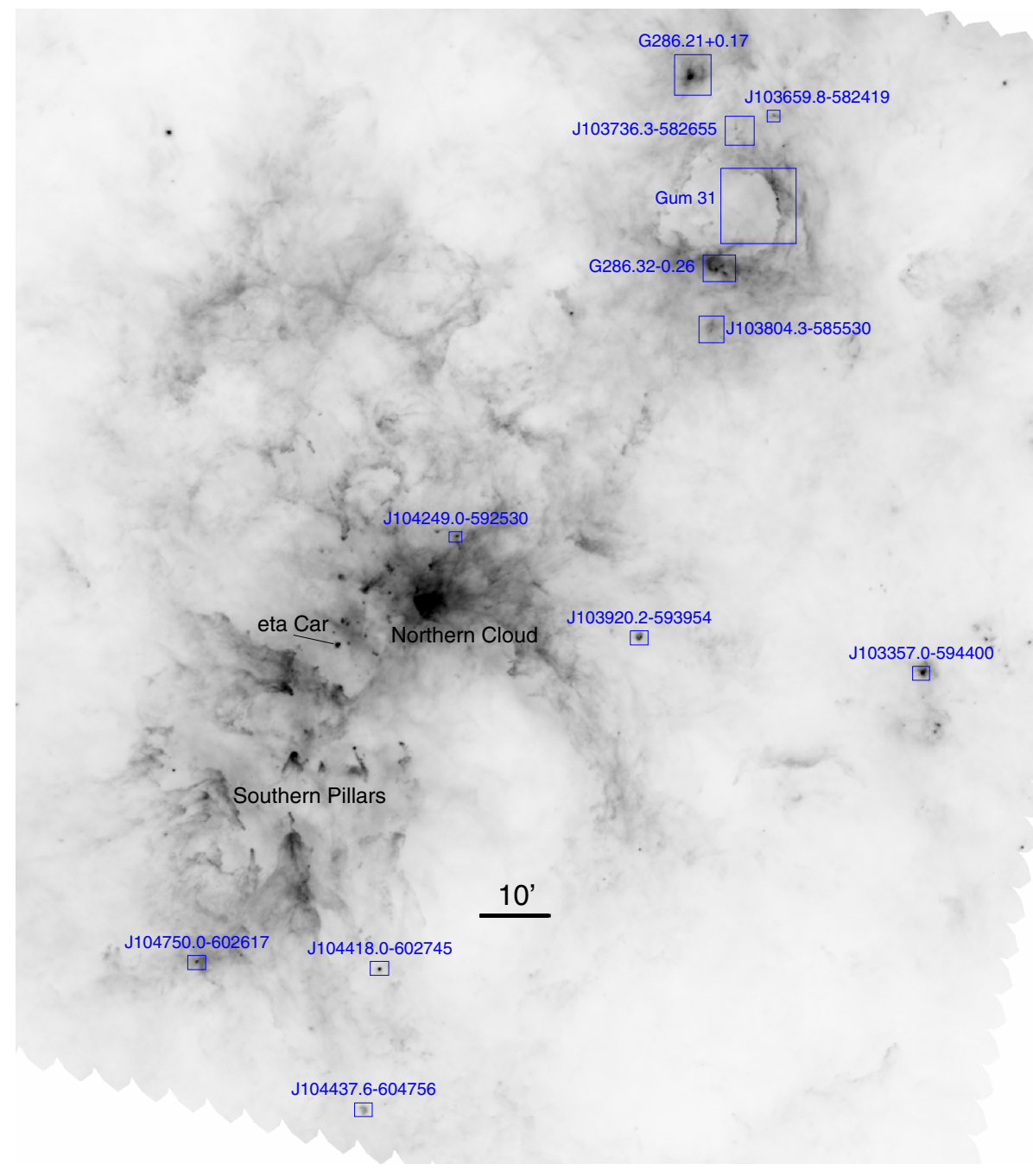

Fig. 5. Herschel $160 \mu \mathrm{m}$ map of the CNC (data from Preibisch et al. 2012). The boxes with labels mark the locations and sizes of all the regions that are shown and discussed in this section (Figs. 6 to 12). North is up and east to the left. infrared sources near and within the inner rim of the dust bubble surrounding the $\mathrm{H}$ II region. The X-ray selected population of young stars in the Gum $31 \mathrm{H}$ II region was recently studied by Preibisch et al. (2014).

\subsubsection{Cluster G286.38-0.26}

Figure 7 shows the VISTA view of the cluster G286.38-0.26, which is partly embedded in a cloud clump in the southern edge of the dust bubble around Gum 31. The bluish and reddish stars trace the different populations of stars seen in front of the clump and embedded in the clump, as discussed in Ohlendorf et al. (2013). A multiwavelength (optical to FIR) comparison of this cluster can be seen in Fig. 12 of Ohlendorf et al. (2013).

\subsubsection{Cluster G286.21+0.17}

Figure 8 shows the VISTA image of the cluster G286.21+0.17. A dense and massive cloud clump (size $\sim 1$ pc, cloud mass $\approx 2100 M_{\odot}$; see Ohlendorf et al. 2013) is located at the southeastern edge of the bright reflection nebulosity that dominates the VISTA image. This clump harbors several deeply embedded young stellar objects, that can only be seen at mid- to farinfrared wavelengths (compare with Fig. 11 in Ohlendorf et al. 2013, for the mid- to far-infrared morphology of this region).
The prominent nebulosity in the VISTA image is illuminated by the stellar cluster at the northwestern edge of the dark cloud.

\subsection{Clusters and groups of embedded objects}

Our visual inspection of the VISTA images revealed a number of interesting objects, for which little information was found in the literature. For the more prominent of these we show here VISTA images and provide a brief description. The numerous very prominent clusters, nebulae, globules, ionization fronts, and cloud pillars in the central regions of the Carina Nebula that have been discussed in the context of previous studies (see Smith et al. 2010a,b; Povich et al. 2011; Preibisch et al. 2011c; Ohlendorf et al. 2013) are excluded from the collection presented here.

All the stellar groups or clusters discussed below are related to prominent FIR cloud features revealed by the Herschel observations of the CNC (see Preibisch et al. 2012). Their locations are marked in Fig. 5. In most cases, the stellar groups appear embedded in, or located close to, prominent dense cloud clumps. In two cases (J103920.2-593954 and J104437.6-604756), stellar clusters are surrounded by prominent ring-like FIR nebulosity (as typical for young clusters which expelled the remnants of their natal clouds).

J103736.3-582655 (Fig. 9): this group is located close to the northern part of the dust bubble around the Gum 31 nebula and 


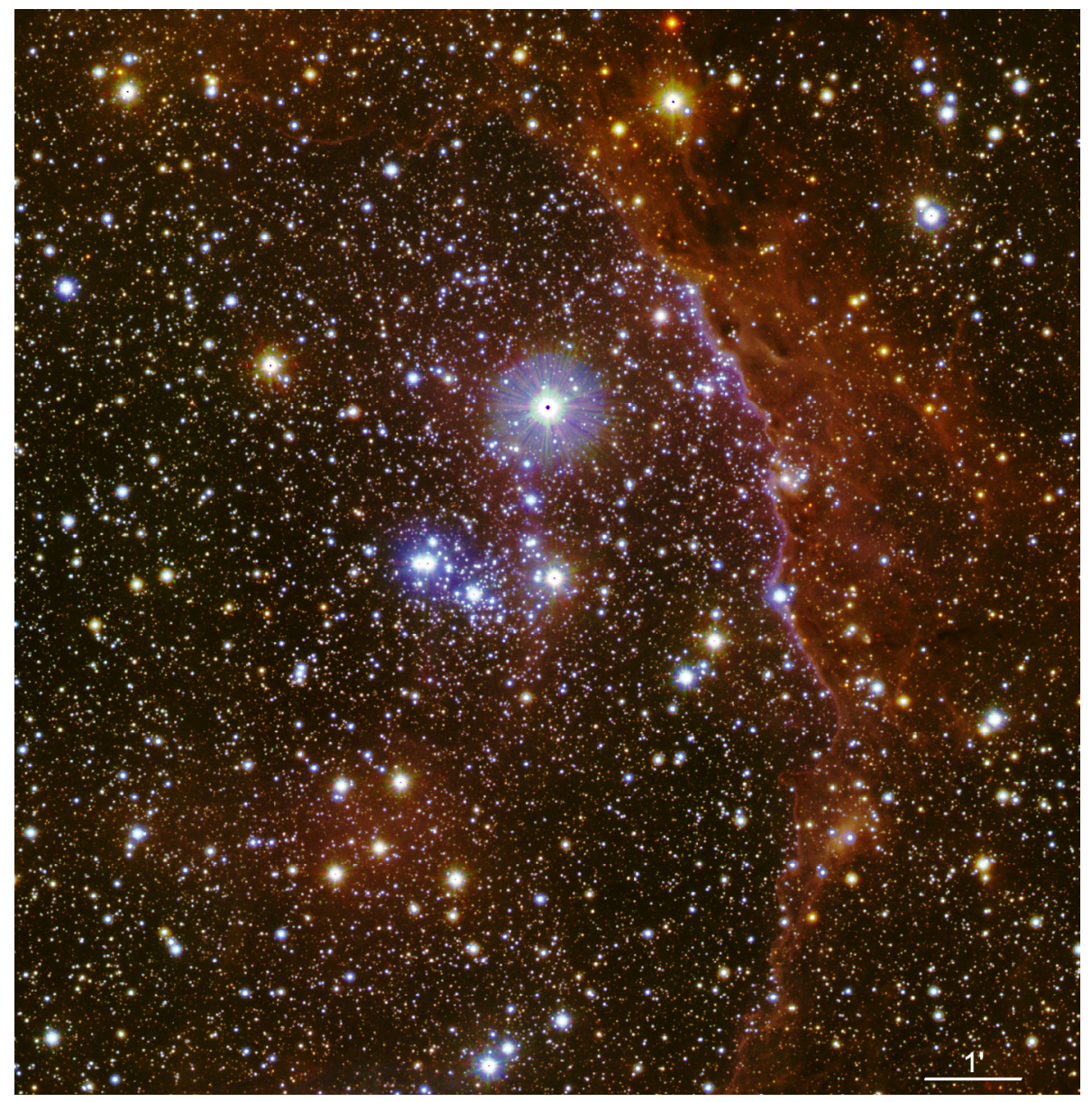

Fig. 6. Three-color composite image constructed from the $J$ - (blue), $H$ - (green), and $K_{\mathrm{s}}$-band (red) VISTA images of the cluster NGC 3324 (near the center) and the western edge of the Gum 31 H II region. North is up and east to the left. The image center is at celestial coordinates $\mathrm{RA}(\mathrm{J} 2000) \approx 10^{\mathrm{h}} 37^{\mathrm{m}} 14^{\mathrm{s}}$, $\operatorname{Dec}(\mathrm{J} 2000)=-58^{\circ} 37^{\prime} 18^{\prime \prime}$.

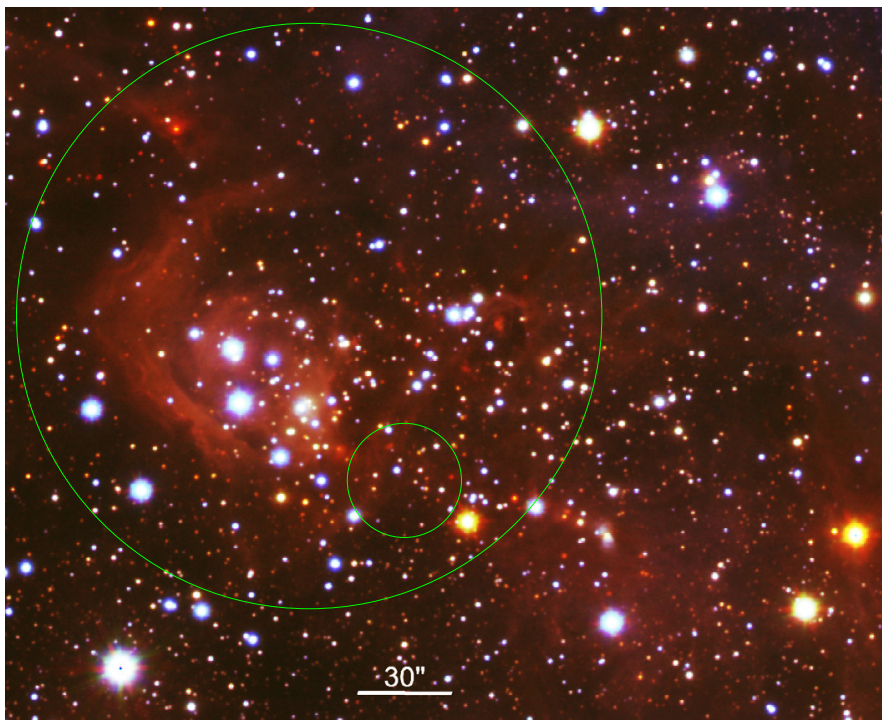

Fig. 7. Three-color composite image constructed from the $J$ - (blue), $H$ - (green), and $K_{\mathrm{s}}$-band (red) VISTA images of the partly embedded cluster G286.38-0.26. North is up and east to the left. The image center is at celestial coordinates $\mathrm{RA}(\mathrm{J} 2000) \approx 10^{\mathrm{h}} 37^{\mathrm{m}} 56^{\mathrm{s}}, \operatorname{Dec}(\mathrm{J} 2000) \approx$ $-58^{\circ} 46^{\prime} 26^{\prime \prime}$. The large green circle marks the approximate location of the $\mathrm{C}^{18} \mathrm{O}$ clump No. 6 , the small green circle that of the $\mathrm{H}^{13} \mathrm{CO}^{+}$clump No. 2 from Yonekura et al. (2005).

is embedded in a prominent dark cloud. Numerous very red, that is, apparently deeply embedded, young stellar objects surrounded by diffuse nebulosity can be seen in the VISTA image.

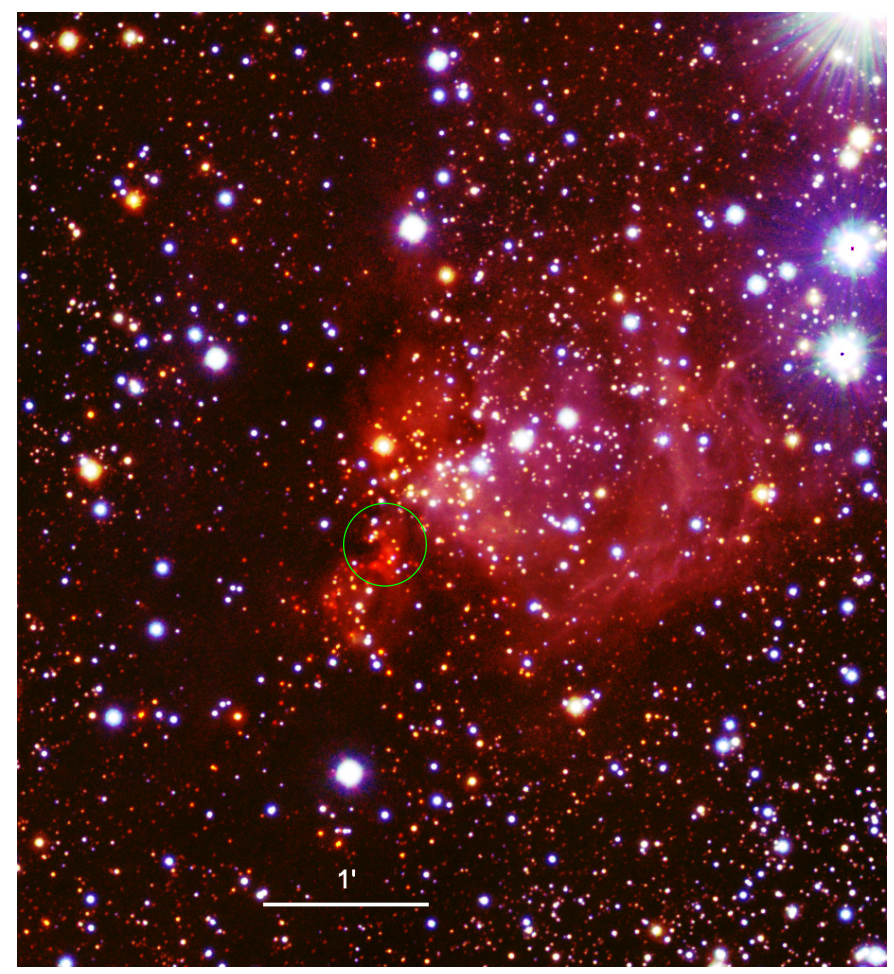

Fig. 8. Three-color composite image constructed from the $J$ - (blue), $H$ (green), and $K_{\mathrm{s}}$-band (red) VISTA images of the cluster G286.21+0.17. North is up and east to the left. The image center is at celestial coordinates $\mathrm{RA}(\mathrm{J} 2000) \approx 10^{\mathrm{h}} 38^{\mathrm{m}} 29^{\mathrm{s}}, \operatorname{Dec}(\mathrm{J} 2000) \approx-58^{\circ} 18^{\prime} 45^{\prime \prime}$. The green circle marks the location of the massive clump mentioned in the text. 


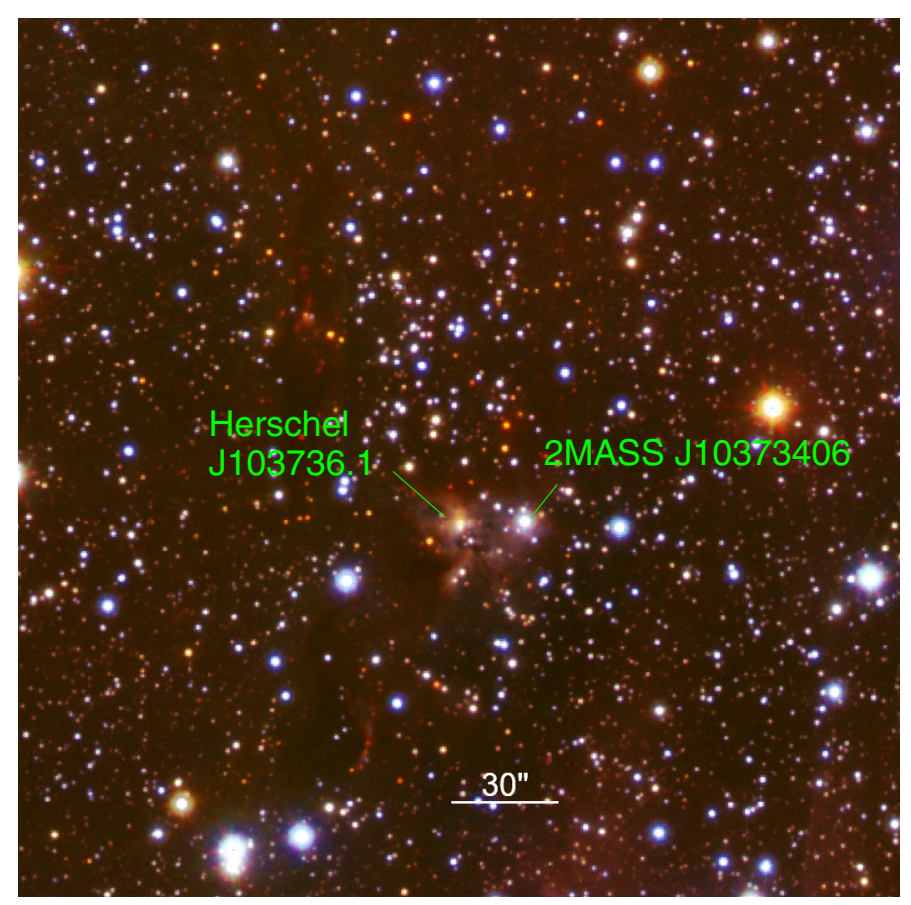

Fig. 9. Three-color composite image constructed from the $J$ - (blue), $H$ - (green), and $K_{\mathrm{s}}$-band (red) VISTA images of the cluster $\mathrm{J} 103736.3-582655$. North is up and east to the left.

The only object in this region listed in SIMBAD is the YSO candidate 2MASS J10373406-5826540, which appears as the bright star just right of and slightly below the center of Fig. 9. The bright source $\approx 20^{\prime \prime}$ east of it, which shows extensive diffuse nebulosity in the VISTA image, coincides with the Herschel source J103736.1-582658 (Gaczkowski et al. 2013), suggesting that this object is in the protostellar phase of evolution.

J103804.3-585530 (Fig. 10): this group is embedded in the $\mathrm{C}^{18} \mathrm{O}$ clump number 7 of Yonekura et al. (2005) that appears as a dark cloud in the VISTA image and contains the FIR source IRAS 10361-5839. Numerous deeply embedded YSOs and diffuse infrared emission can be seen in the VISTA image. The position of the Herschel source J103805.0-585533 (Gaczkowski et al. 2013) coincides with the diffuse red nebulosity seen slightly to the left and below the center of the VISTA image.

J103659.8-582419 (Fig. 11): this is another group of deeply embedded young stellar objects. The brightest NIR source is the YSO candidate 2MASS J10365972-5824186, which shows a very prominent arc-shaped diffuse nebulosity. In its immediate surroundings, several faint red sources can be seen in the VISTA image. The position of the Herschel source J103659.8-582428 (Gaczkowski et al. 2013) coincides with the tip of a triangularshaped elongated reflection nebulosity about $10^{\prime \prime}$ south of of the bright NIR source.

J103357.0-594400 (Fig. 12, top left): this group of objects is embedded in a prominent clump seen in the Herschel maps. The position and approximate extent of the compact FIR emission is marked by the circle. The brightest source in the VISTA image (close to the crossing of the coordinate lines) can be identified with IRAS 10320-5928 = MSX6C G286.3938-01.3514,

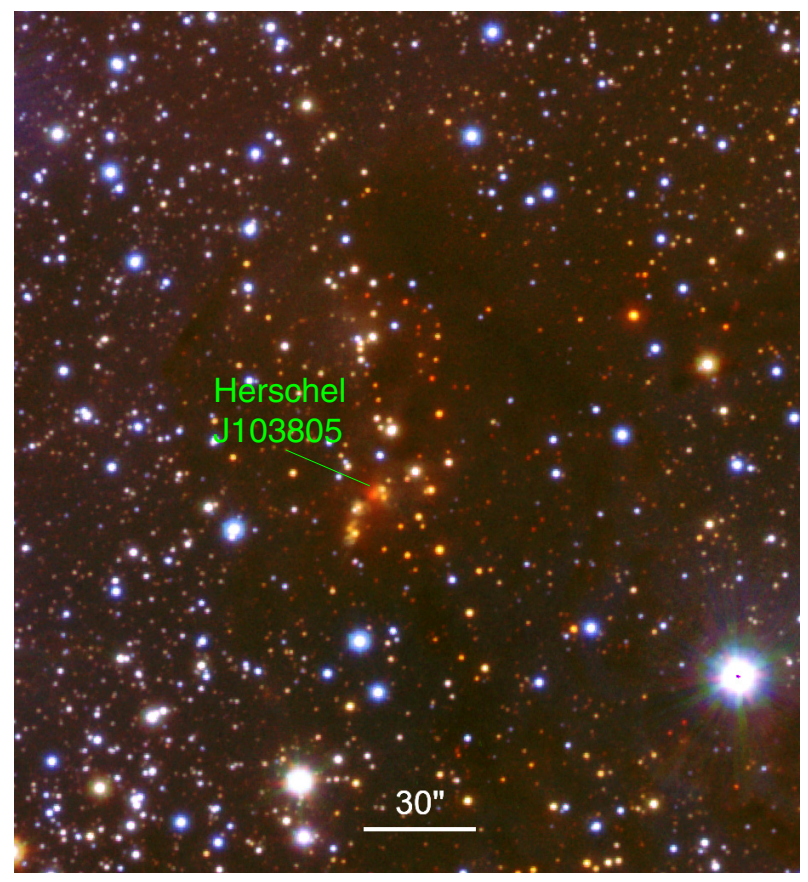

Fig. 10. Three-color composite image constructed from the $J$ - (blue), $H$ - (green), and $K_{\mathrm{s}}$-band (red) VISTA images of the cluster J103804.3-585530. North is up and east to the left.

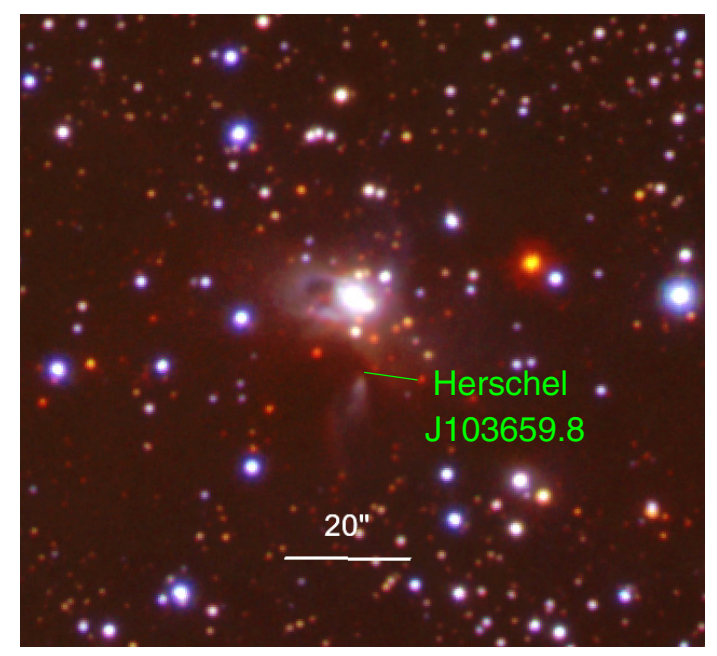

Fig. 11. Three-color composite image constructed from the $J$ - (blue), $H$ - (green), and $K_{\mathrm{s}}$-band (red) VISTA images of the cluster J103659.8582419. North is up and east to the left.

which contains two massive YSO candidates (see Mottram et al. 2007). In the Herschel data, the corresponding point source J103356.5-594358 is one of the brightest FIR sources in the whole area of the CNC Herschel map (Gaczkowski et al. 2013). Since this group of objects is located at the periphery of our VISTA survey area and the surrounding FIR emission appears to be clearly separated from the nebulosity of the Carina Nebula (see Fig. 5), these clouds (and their embedded objects) are probably unrelated to the Carina Nebula and are part of the background cloud complex G286.4-1.3 with an estimated distance of $\sim 9 \mathrm{kpc}$ (see the discussion in Roccatagliata et al. 2013).

J103920.2-593954 (Fig. 12, top middle): this group of objects clusters around the FIR source IRAS 10374-5924 and is 

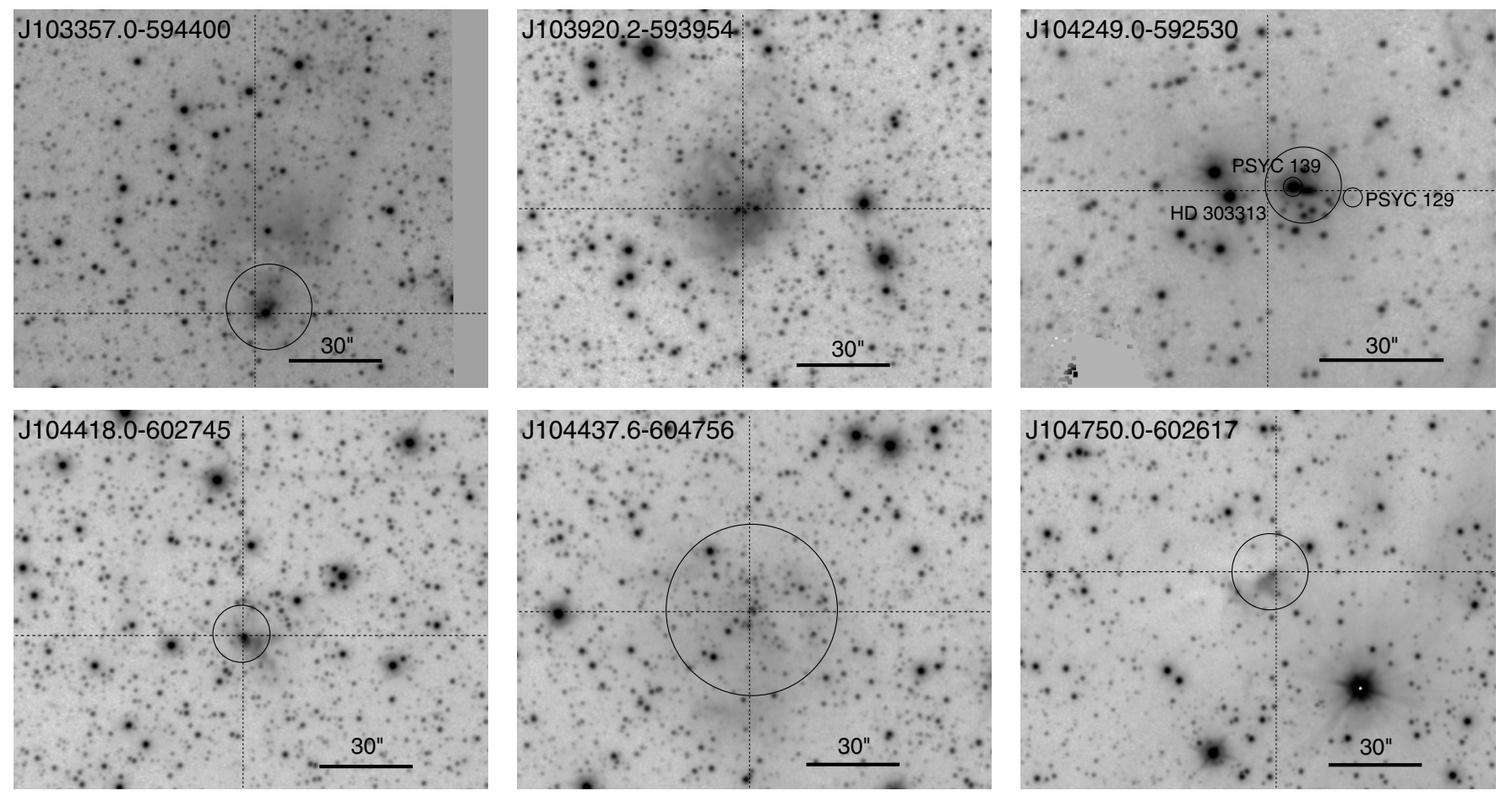

Fig. 12. Negative grayscale representation of $K_{\mathrm{s}}$-band VISTA images of selected clusters in the area. A logarithmic intensity scale is used to show the faint stars and weak nebulosity. North is up and east to the left. The vertical and horizontal lines show the right ascension and declination as given in the upper left corner of each image. The circles mark the position of the cloud clumps and objects as described in the text.

surrounded by strong diffuse nebulosity in the VISTA image. The Herschel maps (see Preibisch et al. 2012) show a prominent ring-like nebulosity with a diameter of $\sim 45^{\prime \prime}$ around this cluster. This cluster is located about $40^{\prime}$ west of the center of the Carina Nebula, that is, at a projected distance of $\sim 25 \mathrm{pc}$. It may thus be a background object and not part of the CNC.

J104249.0-592530 (Fig. 12, top right): this group of stars is embedded in the $\mathrm{C}^{18} \mathrm{O}$ clump number 9 of Yonekura et al. (2005), which corresponds to a bright and compact FIR clump containing the Herschel source J104248.0-592530 (Gaczkowski et al. 2013), the position of which is marked by the large circle in the figure. The clump is located at the northern edge of the so-called Northern Cloud (see Preibisch et al. 2012), which constitutes the FIR-brightest and most massive cloud structure in the entire CNC. The group of stars visible in the VISTA image includes the B1.5 star HD 303313 and the young stellar objects PCYC 129 and PCYC 139 (Povich et al. 2011), both of which are marked by small circles in the figure. The nominal position of the mid-infrared source MSX6C G287.22-00.53 (which is classified as an H II region; see Rathborne et al. 2004) corresponds to the crossing of the coordinate lines at the center of the clustering.

J104418.0-602745 (Fig. 12, bottom left): this group of stars is associated with the very bright Herschel point-like source J104418.1-602744 (Gaczkowski et al. 2013) at the southern periphery of the CNC. The circle marks the position of this Herschel source. The NIR point source at the crossing of the coordinate lines is 2MASX J10441798-6027447, for which a spectral type of B0 is listed in SIMBAD. This object coincides very well with the Herschel source position and shows a prominent unipolar triangular nebula that seems to be a reflection nebulosity in the envelope of an embedded YSO. The corresponding mid-infrared source G287.8768-01.3618 is listed as a massive YSO candidate in Mottram et al. (2007).

J104437.6-604756 (Fig. 12, bottom middle): this faint, but populous cluster of stars is located at the southern edge of the VCNS field; it may be a background object that is unrelated to the CNC. In the Herschel maps (see Preibisch et al. 2012), this cluster is surrounded by a prominent arc-like nebulosity with a diameter of about one arcminute, the extent of which is approximately delineated by the circle. The cluster coincides with the IRAS source 10427-6032, which is classified as an H II region (Kerber et al. 2000).

J104750.0-602617 (Fig. 12, bottom right): this small group of objects appears to be embedded in the $\mathrm{C}^{18} \mathrm{O}$ clump number 15 of Yonekura et al. (2005), which is located in the Southern Pillars region of the CNC. The circle shows the approximate extent of the compact FIR emission seen in the Herschel maps. Near the center of this clump at the crossing of the coordinate lines in the figure, a prominent triangular-shaped nebula can be seen. The tip position of the nebula coincides with the Herschel point-like source J104750.7-602619 (Gaczkowski et al. 2013) and the mid-infrared source PCYC 1317. This object belongs to cluster O identified by Smith et al. (2010b) in their analysis of the Spitzer maps.

\section{Summary}

The VISTA Carina Nebula Survey (VCNS) provides, for the first time, NIR imaging data with sufficient sensitivity to detect the full low-mass stellar population associated with the Carina 
Table A.1. Stars with particularly high proper motion in the VCNS catalog.

\begin{tabular}{|c|c|c|c|c|c|c|c|c|c|}
\hline 2MASS name & $\begin{array}{l}\alpha_{\text {VISTA }} \\
{[\mathrm{J} 2000]}\end{array}$ & $\begin{array}{l}\delta_{\text {VISTA }} \\
{[\mathrm{J} 2000]}\end{array}$ & $\begin{array}{r}J \\
{[\mathrm{mag}]}\end{array}$ & $\begin{array}{r}H \\
{[\mathrm{mag}]}\end{array}$ & $\begin{array}{r}K_{\mathrm{s}} \\
{[\mathrm{mag}]}\end{array}$ & $\begin{array}{r}\mu_{\alpha} \\
{[\mathrm{mas} / \mathrm{yr}]}\end{array}$ & $\begin{array}{r}\mu_{\delta} \\
{[\mathrm{mas} / \mathrm{yr}]}\end{array}$ & $\begin{array}{r}\mu_{\alpha} \\
(\mathrm{SIMBAD})\end{array}$ & $\begin{array}{r}\mu_{\delta} \\
(\mathrm{SIMBAD})\end{array}$ \\
\hline $\mathrm{J} 10335803-6037459$ & $10: 33: 57.87$ & $-60: 37: 46.0$ & 11.93 & 11.28 & 11.14 & -95 & 2 & & \\
\hline J10341811-5830102 & 10:34:18.06 & $-58: 30: 11.6$ & 12.31 & 11.69 & 11.32 & -34 & -105 & & \\
\hline J10343281-5826345 & $10: 34: 32.90$ & $-58: 26: 35.3$ & 10.38 & 9.75 & 9.58 & 56 & -67 & & \\
\hline J10345713-5816511 & $10: 34: 57.01$ & $-58: 16: 50.8$ & 10.16 & 9.95 & 9.90 & -81 & 30 & & \\
\hline J10351573-5814080 & $10: 35: 15.49$ & $-58: 14: 07.6$ & 9.98 & 9.37 & 9.15 & -158 & 33 & & \\
\hline J10352386-5801512 & $10: 35: 23.81$ & $-58: 01: 50.0$ & 10.09 & 9.76 & 9.69 & -32 & 107 & -33.4 & 110.9 \\
\hline J10380859-5847094 & $10: 38: 08.36$ & $-58: 47: 08.5$ & 10.61 & 10.37 & 10.34 & -156 & 85 & -161.0 & 91.3 \\
\hline J10391074-6006413 & 10:39:10.61 & $-60: 06: 40.5$ & 9.72 & 9.41 & 9.41 & -88 & 72 & -96.6 & 60.5 \\
\hline J10391101-5947139 & $10: 39: 11.19$ & $-59: 47: 14.7$ & 11.98 & 11.38 & 11.13 & 111 & -57 & & \\
\hline J10410762-5958195 & $10: 41: 07.49$ & $-59: 58: 19.6$ & 11.75 & 11.04 & 10.91 & -85 & -1 & & \\
\hline J10413241-5807078 & $10: 41: 32.20$ & $-58: 07: 07.2$ & 11.75 & 11.15 & 10.89 & -142 & 56 & & \\
\hline J10424734-6002394 & $10: 42: 46.75$ & $-60: 02: 36.6$ & 7.37 & 6.99 & 6.94 & -366 & 241 & -313.0 & 198.4 \\
\hline J10431886-6012075 & $10: 43: 18.72$ & $-60: 12: 08.3$ & 8.32 & 7.54 & 7.28 & -84 & -60 & & \\
\hline J10440265-5812256 & 10:44:02.71 & $-58: 12: 26.6$ & 9.59 & 8.95 & 8.72 & 36 & -80 & & \\
\hline $\mathrm{J} 10444003-5810278$ & $10: 44: 39.83$ & $-58: 10: 24.7$ & 8.68 & 8.08 & 7.96 & -140 & 263 & -143.06 & 247.55 \\
\hline J10452889-5802225 & $10: 45: 28.76$ & $-58: 02: 22.1$ & 10.27 & 9.67 & 9.61 & -87 & 32 & & \\
\hline J10465495-5836316 & $10: 46: 54.74$ & $-58: 36: 30.7$ & 11.89 & 11.26 & 11.05 & -141 & 82 & & \\
\hline $\mathrm{J} 10471349-6027228$ & $10: 47: 13.32$ & $-60: 27: 22.6$ & 9.95 & 9.56 & 9.50 & -110 & 17 & -105.2 & 21.3 \\
\hline $\mathrm{J} 10482132-6028070$ & $10: 48: 21.11$ & $-60: 28: 06.6$ & 11.78 & 11.13 & 10.89 & -134 & 30 & & \\
\hline J10485248-6029354 & $10: 48: 52.30$ & $-60: 29: 35.1$ & 10.53 & 10.04 & 9.98 & -113 & 29 & & \\
\hline J10490202-5854011 & 10:49:01.65 & $-58: 54: 02.4$ & 11.55 & 10.98 & 10.70 & -242 & -104 & & \\
\hline J10491609-5833033 & 10:49:16.31 & $-58: 33: 04.3$ & 11.58 & 10.98 & 10.69 & 137 & -80 & & \\
\hline J10503017-5906260 & 10:50:30.07 & $-59: 06: 27.1$ & 11.38 & 10.84 & 10.56 & -67 & -88 & & \\
\hline J10504937-5756334 & $10: 50: 49.24$ & $-57: 56: 34.4$ & 11.41 & 10.83 & 10.68 & -87 & -74 & & \\
\hline J10510582-6040139 & 10:51:05.69 & $-60: 40: 13.8$ & 11.89 & 11.14 & 11.01 & -83 & 11 & & \\
\hline
\end{tabular}

Notes. We list the 2MASS point source catalog designation (that contains the 2MASS J2000 coordinates), the celestial coordinates determined in our VISTA $H$-band data, the 2MASS catalog magnitudes, the calculated proper motions, and the proper motions listed in SIMBAD (if available).

Nebula across the entire $\sim 5$ square-degree angular extent of this star-forming complex.

We here described the motivation of the survey and presented the observations and data analysis. We also characterized the data. The catalog of nearly four million reliably detected objects within the 6.7 square-degree survey area is made available at the CDS. We presented a collection of VISTA images of young stellar groups and clusters in the outer parts of the CNC that have not been studied in detail before.

The VCNS images and the source catalog provide a new basis for spatially complete studies of the stellar populations in the CNC. These will be the topics of forthcoming publications.

Acknowledgements. This work is based on observations made with ESO telescopes at the La Silla Paranal Observatory under program ID 088.C-0117(A). We gratefully acknowledge funding for this project by the German Deutsche Forschungsgemeinschaft, DFG project number PR 569/9-1. Additional support came from funds from the Munich Cluster of Excellence: "Origin and Structure of the Universe". This publication makes use of data products from the Two Micron All Sky Survey, which is a joint project of the University of Massachusetts and the Infrared Processing and Analysis Center/California Institute of Technology, funded by the National Aeronautics and Space Administration and the National Science Foundation. This research has made use of the SIMBAD database, operated at CDS, Strasbourg, France, and the Digitized Sky Surveys, that were produced at the Space Telescope Science Institute under US Government grant NAG W-2166.

\section{Appendix A: Objects with high proper motion}

In our analysis we noticed several bright stars in the VISTA images that did not match a 2 MASS catalog object within a $0.5^{\prime \prime}$ radius, but for which a 2 MASS point source catalog object of the expected magnitude was found at a slightly larger angular offset. This indicates that the positions of these stars have noticeably

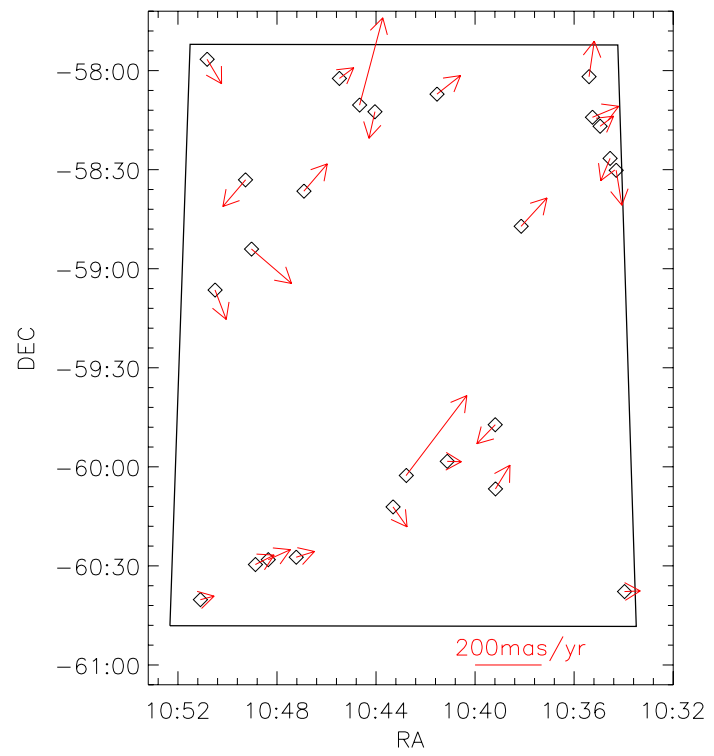

Fig. A.1. Spatial distribution of objects with a high proper motion in the VCNS area. The arrows indicate the proper-motion vectors of these objects (scaled by a factor of 500 with respect to the coordinate system).

changed between the 2MASS and the VISTA observation. We visually inspected the VISTA and 2MASS images in detail to ensure that the identification is correct and found 25 objects that obviously show high proper motions.

To calculate the proper-motion vectors from our VISTA positions and the 2MASS positions, we assumed a time base 


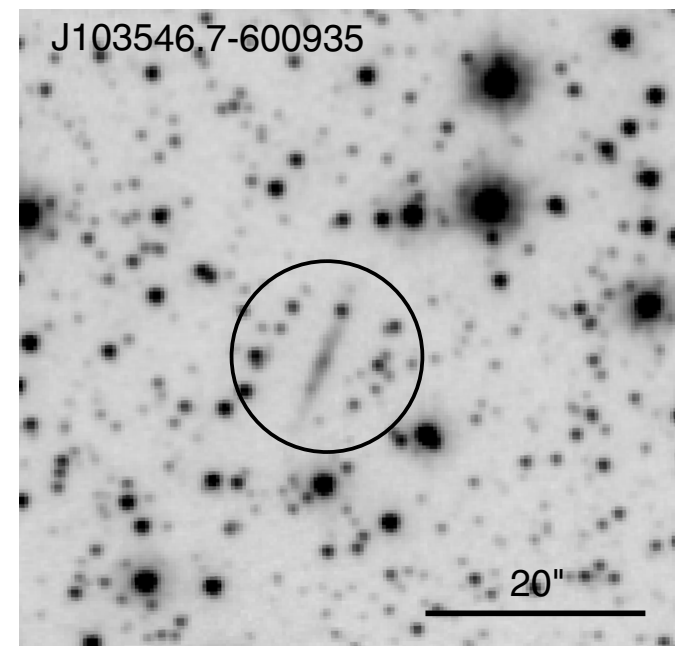

Fig. B.1. Negative grayscale representation of the VISTA $H$-band image of the edge-on galaxy candidate J103546.7-600935. North is up and east to the left.

of 12 years and 1 month $^{9}$. Considering the uncertainties in the astrometry of VISTA ( 60 mas) and 2MASS ( $\leq 100$ mas) and the period of about two weeks during which the VISTA images were obtained, the uncertainties of the derived proper motions are about 9-15 mas/yr. In Table A.1, these objects with a high proper motion are listed. Our proper-motion measurements agree well with the proper-motions listed in SIMBAD, where available. The spatial distribution objects with a high proper motion in the VCNS area and their proper-motion vectors are shown in Fig. A.1.

\section{Appendix B: Serendipitous detection of an edge-on galaxy candidate}

At the southwestern periphery of the Carina Nebula Cloud, at a position with low cloud column density, we discovered a faint, strongly elongated object in our VISTA images. This object shows the typical morphology of an edge-on galaxy; it is marked by the circle in Fig. B.1, and we denote it as J103546.7-600935. It is neither visible in the Digitized Sky Survey optical images, nor in the Herschel maps and is not listed in SIMBAD.

\section{References}

Ascenso, J., Alves, J., Vicente, S., \& Lago, M. T. V. T. 2007, A\&A, 476, 199 Baraffe, I., Chabrier, G., Allard, F., \& Hauschildt, P. H. 1998, A\&A, 337, 403
Briceño, C., Preibisch, Th., Sherry, W., et al. 2007, in Protostars \& Planets V, eds. B. Reipurth, D. Jewitt, \& K. Keil (Tucson: University of Arizona Press), 345

Broos, P. S., Townsley, L. K., Feigelson, E. D., et al. 2011a, ApJS, 194, 2

Broos, P. S., Getman, K.V., Povich, M.S., et al. 2011b, ApJS, 194, 4

Dalton, G. B., Caldwell, M., Ward, A. K., et al. 2006, Proc. SPIE, 6269, 62690X

Dias, W. S., Alessi, B. S., Moitinho, A., \& Lépine, J. R. D. 2002, A\&A, 389, 871

Draine, B. T. 2011, Physics of the Interstellar and Intergalactic Medium (Princeton University Press)

Emerson, J., McPherson, A., \& Sutherland, W. 2006, The Messenger, 126, 41

Evans, C. J., Smartt, S. J., Lee, J.-K., et al. 2005, A\&A, 437, 467

Feigelson, E. D., \& Babu, G. J. 2012, Modern Statistical Methods for Astronomy (Cambridge University Press)

Feigelson, E. D., Getman, K. V., Townsley, L. K., et al. 2011, ApJS, 194, 9

Gaczkowski, B., Preibisch, T., Ratzka, T., et al. 2013, A\&A, 549, A67

Getman, K. V., Feigelson, E. D., Kuhn, M. A., et al. 2014, ApJ, 787, 108

Gum, C. S. 1955, MmRAS, 67, 155

Herschel, J. F. W., Sir 1847, Results of astronomical observations made during the years $1834,5,6,7,8$, at the Cape of Good Hope; being the completion of a telescopic survey of the whole surface of the visible heavens, commenced in 1825 (London: Smith, Elder and co)

Hodgkin, S. T., Irwin, M. J., Hewett, P. C., \& Warren, S. J. 2009, MNRAS, 394, 675

Irwin, M. J., Lewis, J., Hodgkin, S., et al. 2004, Proc. SPIE, 5493, 411

Kerber, F., Furlan, E., Roth, M., Galaz, G., \& Chanamé, J. C. 2000, PASP, 112, 542

Lejeune T., \& Schaerer, D. 2001, A\&A, 366, 538

Maíz-Apellániz, J., Walborn, N. R., Galué, H. Á., \& Wei, L. H. 2004, ApJS, 151, 103

Mottram, J. C., Hoare, M. G., Lumsden, S. L., et al. 2007, A\&A, 476, 1019

Ohlendorf, H., Preibisch, T., Gaczkowski, B., et al. 2013, A\&A, 552, A14

Pilbratt, G. L., Riedinger, J. R., Passvogel, T., et al. 2010, A\&A, 518, L1

Povich, M. S., Smith, N., Majewski, S. R., et al. 2011, ApJS, 194, 14

Preibisch, T., Schuller, F., Ohlendorf, H., et al. 2011a, A\&A, 525, A92

Preibisch, T., Hodgkin, S., Irwin, M., et al. 2011b, ApJS, 194, 10

Preibisch, T., Ratzka, T., Kuderna, B., et al. 2011c, A\&A, 530, A34

Preibisch, T., Roccatagliata, V., Gaczkowski, B., \& Ratzka, T. 2012, A\&A, 541, A132

Preibisch, T., Mehlhorn, M., Townsley, L., Broos, P., \& Ratzka, T. 2014, A\&A, 564, A120

Rathborne, J. M., Brooks, K. J., Burton, M. G., Cohen, M., \& Bontemps, S. 2004, A\&A, 418, 563

Roccatagliata, V., Preibisch, T., Ratzka, T., \& Gaczkowski, B. 2013, A\&A, 554, A6

Sana, H., Momany, Y., Gieles, M., et al. 2010, A\&A, 515, A26

Siess, L., Dufour, E., \& Forestini, M. 2000, A\&A, 358, 593

Skrutskie, M. F., Cutri, R. M., Stiening, R., et al. 2006, AJ, 131, 1163

Smith, N. 2006, MNRAS, 367, 763

Smith, N., \& Brooks, K. J. 2007, MNRAS, 379, 1279

Smith, N., \& Brooks, K. J. 2008, in Handbook of Star Forming Regions, Vol. II:

The Southern Sky, ASP Monograph Publications (ed. Bo Reipurth), 5, 138

Smith, N., Egan, M. P., Carey, S., et al. 2000, ApJ, 532, L145

Smith, N., Bally, J., \& Walborn, N. R. 2010a, MNRAS, 405, 1153

Smith, N., Povich, M. S., Whitney, B. A., et al. 2010b, MNRAS, 406, 952

Soto, M., Barbá, R., Gunthardt, G., et al. 2013, A\&A, 552, A101

Townsley, L. K., Broos, P. S., Corcoran, M. F., et al. 2011, ApJS, 194, 1

Wang, J., Feigelson, E. D., Townsley, L. K., et al. 2011, ApJS, 194, 11

Wolk, S. J., Broos, P. S., Getman, K. V., et al. 2011, ApJS, 194, 12

Yonekura, Y., Asayama, S., Kimura, K., et al. 2005, ApJ 634, 476

\footnotetext{
9 The 2MASS images were obtained at the end of January 2000, while our VISTA images were obtained between 8 and 24 March 2012.
} 